\title{
Existence Results for Impulsive Fractional $q$-Difference Equation with Antiperiodic Boundary Conditions
}

\author{
Mingyue Zuo (D) and Xinan Hao \\ School of Mathematical Sciences, Qufu Normal University, Qufu 273165, Shandong, China \\ Correspondence should be addressed to Xinan Hao; haoxinan2004@163.com
}

Received 17 July 2018; Accepted 17 September 2018; Published 9 October 2018

Academic Editor: Maria Alessandra Ragusa

Copyright ( 2018 Mingyue Zuo and Xinan Hao. This is an open access article distributed under the Creative Commons Attribution License, which permits unrestricted use, distribution, and reproduction in any medium, provided the original work is properly cited.

In this paper, we investigate the impulsive fractional $q$-difference equation with antiperiodic conditions. The existence and uniqueness results of solutions are established via the theorem of nonlinear alternative of Leray-Schauder type and the Banach contraction mapping principle. Two examples are given to illustrate our results.

\section{Introduction}

In this paper, we are concerned with the existence and uniqueness of solutions for the following impulsive fractional $q$-difference equation with antiperiodic boundary conditions

$$
\begin{aligned}
{ }^{c} D_{q}^{\alpha} u(t) & =f(t, u(t), T u(t), S u(t)), \\
t \in J^{\prime}=J \backslash\left\{t_{1}, t_{2}, \ldots, t_{m}\right\}, & \quad k=1,2, \ldots, m, \\
\left.\Delta u\right|_{t=t_{k}} & =I_{k}\left(u\left(t_{k}^{-}\right)\right), \\
\left.\Delta D_{q} u\right|_{t=t_{k}} & =I_{k}^{*}\left(u\left(t_{k}^{-}\right)\right), \\
u(0) & =-u(1), \\
{ }^{c} D_{q}^{\beta} u(0) & =-{ }^{c} D_{q}^{\beta} u(1),
\end{aligned}
$$

where $q \in(0,1), 1<\alpha \leq 2,0<\beta<1, \alpha-\beta-1>0$, $J=[0,1], D_{q}$ is $q$-derivative, ${ }^{c} D_{q}^{\alpha}$, and ${ }^{c} D_{q}^{\beta}$ denote the Caputo $q$-derivative of orders $\alpha$ and $\beta$, respectively. $f \in C(J \times \mathbb{R} \times \mathbb{R} \times$ $\mathbb{R}, \mathbb{R}), I_{k}, I_{k}^{*} \in C(\mathbb{R}, \mathbb{R})(k=1,2, \ldots, m), \mathbb{R}$ is the set of all real numbers, and $0=t_{0}<t_{1}<\cdots<t_{m}<t_{m+1}=1 . T$ and $S$ are linear operators defined by

$$
\begin{aligned}
& T u(t)=\int_{0}^{t} k(t, s) u(s) d_{q} s, \\
& \operatorname{Su}(t)=\int_{0}^{1} h(t, s) u(s) d_{q} s,
\end{aligned}
$$

$$
t \in J \text {, }
$$

where $k \in C(D, \mathbb{R}), h \in C(J \times J, \mathbb{R}), D=\{(t, s) \in J \times J: t \geq s\}$. $\left.\Delta u\right|_{t=t_{k}}=u\left(t_{k}^{+}\right)-u\left(t_{k}^{-}\right)$, where $u\left(t_{k}^{+}\right)$and $u\left(t_{k}^{-}\right)$represent the right and left limits of $u(t)$ at $t=t_{k},\left.\Delta D_{q} u\right|_{t=t_{k}}=D_{q} u\left(t_{k}^{+}\right)-$ $D_{q} u\left(t_{k}^{-}\right)$has a similar meaning.

Fractional $q$-difference calculus plays a very important role in modern applied mathematics due to their deep physical background and has been studied extensively [14]. Impulsive differential equations are important in both theory and applications. Considerable effort has been devoted to differential equations with or without impulse, for example, [5-21]. In recent years, impulsive fractional difference and differential equations with antiperiodic conditions have received much attention; see [22-27] and the references therein. Zhang and Wang [24] have applied cone contraction fixed point theorem to establish the existence of solutions to nonlinear fractional differential equation with impulses and antiperiodic boundary conditions

$$
\begin{aligned}
{ }^{c} D^{\alpha} u(t) & =f(t, u(t)), \\
1 & <\alpha \leq 2, t \in J \backslash\left\{t_{1}, t_{2}, \ldots, t_{m}\right\}, J=[0, T], \\
\left.\Delta u\right|_{t=t_{k}}= & I_{k}\left(u\left(t_{k}\right)\right),
\end{aligned}
$$




$$
\begin{gathered}
\left.\Delta u^{\prime}\right|_{t=t_{k}}=I_{k}^{*}\left(u\left(t_{k}\right)\right), \\
u(0)=-u(T), \\
u^{\prime}(0)=-u^{\prime}(T),
\end{gathered}
$$$$
k=1,2, \ldots, p
$$

where ${ }^{c} D^{\alpha}$ is the Caputo fractional derivative, $f \in C(J \times$ $\mathbb{R}, \mathbb{R}), I_{k}, I_{k}^{*} \in C(\mathbb{R}, \mathbb{R})$. By using Banach fixed point theorem, Schaefer fixed point theorem, and nonlinear alternative of Leray-Schauder type theorem, some existence results of solutions for problem (3) are obtained in [25]. Ahmad et al. [28] studied existence of solutions for the following antiperiodic boundary value problem (BVP for short) of impulsive fractional $q$-difference equation

$$
\begin{aligned}
& { }_{t_{k}}^{c} D_{q_{k}}^{\alpha_{k}} x(t)=f(t, x(t)), \quad t \in J_{k} \subseteq[0, T], t \neq t_{k}, \\
& \left.\Delta x\right|_{t=t_{k}}=x\left(t_{k}^{+}\right)-x\left(t_{k}\right)=\varphi_{k}\left({ }_{t_{k-1}} I_{q_{k-1}}^{\beta_{k-1}} x\left(t_{k}\right)\right), \\
& k=1,2, \ldots, m, \\
& { }_{t_{k}} D_{q_{k}} x\left(t_{k}^{+}\right)-{ }_{t_{k-1}} D_{q_{k-1}} x\left(t_{k}\right)=\varphi_{k}^{*}\left({ }_{t_{k-1}} I_{q_{k-1}}^{\gamma_{k-1}} x\left(t_{k}\right)\right), \\
& k=1,2, \ldots, m, \\
& x(0)=-x(T), \\
& { }_{0} D_{q 0} x(0)=-{ }_{t_{m}} D_{q_{m}} x(T),
\end{aligned}
$$

where ${ }_{t_{k}}^{c} D_{q_{k}}^{\alpha_{k}}$ denotes the Caputo $q_{k}$-fractional derivative of order $\alpha_{k}$ on $J_{k}, 1<\alpha_{k} \leq 2,0<q_{k}<1, f \in C(J \times$ $\mathbb{R}, \mathbb{R}), \varphi_{k}, \varphi_{k}^{*} \in C(\mathbb{R}, \mathbb{R}), k=1,2, \ldots, m \cdot{ }_{t_{k}} I_{q_{k}}^{\beta_{k}}$ and ${ }_{t_{k}} I_{q_{k}}^{\gamma_{k}}$ denote the Riemann-Liouville $q_{k}$-integral of orders $\beta_{k}$ and $\gamma_{k}$, respectively.

In this paper we are concerned with the existence and uniqueness of solutions for impulsive fractional $q$-difference equation antiperiodic BVP. By applying the theorem of nonlinear alternative of Leray-Schauder type and Banach contraction mapping principle, we show the existence and uniqueness of solutions for the BVP (1). Some ideas of this paper are from $[29,30]$.

\section{Preliminaries and Lemmas}

For $q \in(0,1)$, let

$$
\begin{aligned}
{[a]_{q} } & =\frac{1-q^{a}}{1-q}, \\
(a ; q)_{\infty} & =\prod_{i=0}^{\infty}\left(1-a q^{i}\right), \\
(a ; q)_{\alpha} & =\frac{(a ; q)_{\infty}}{\left(a q^{\alpha} ; q\right)_{\infty}},
\end{aligned}
$$

$(a, \alpha \in \mathbb{R})$.
We define the $q$-analogue of the power function $(a-b)^{n}$ with $n \in \mathbb{N}_{0}$ is

$$
\begin{aligned}
& (a-b)^{0}=1, \\
& (a-b)^{n}=\prod_{k=0}^{n-1}\left(a-b q^{k}\right),
\end{aligned}
$$

$n \in \mathbb{N}, a, b \in \mathbb{R}$

and, for $\alpha \in \mathbb{R}$,

$$
(a-b)^{(\alpha)}=a^{\alpha} \prod_{n=0}^{\infty} \frac{a-b q^{n}}{a-b q^{\alpha+n}} .
$$

The $q$-derivative of $f$ is defined by

$$
\begin{aligned}
& \left(D_{q} f\right)(x)=\frac{f(x)-f(q x)}{(1-q) x}, \\
& \left(D_{q} f\right)(0)=\lim _{x \rightarrow 0}\left(D_{q} f\right)(x)
\end{aligned}
$$

and $q$-derivative of higher order by

$$
\begin{aligned}
& \left(D_{q}^{0} f\right)(x)=f(x), \\
& \left(D_{q}^{n} f\right)(x)=D_{q}\left(D_{q}^{n-1} f\right)(x), \quad n \in \mathbb{N} .
\end{aligned}
$$

The $q$-integral of $f$ is defined by

$$
\left(I_{q} f\right)=\int_{0}^{x} f(t) d_{q} t=x(1-q) \sum_{n=0}^{\infty} f\left(x q^{n}\right) q^{n},
$$

$$
x \in[0, b] .
$$

Lemma 1 (see [31]). (1) If $|f|$ is q-integral on the interval [0, $x]$, then $\left|\int_{0}^{x} f(t) d_{q} t\right| \leq \int_{0}^{x}|f(t)| d_{q} t$.

(2) If $f$ and $g$ are $q$-integral on the interval $[0, x], f(t) \leq$ $g(t)$ for all $t \in[0, x]$, then $\int_{0}^{x} f(t) d_{q} t \leq \int_{0}^{x} g(t) d_{q} t$.

Definition 2 (see [2]). Let $\alpha \geq 0$ and $f$ be a function defined on $[0, b]$. The fractional $q$-integral of the Riemann-Liouville type is defined by $\left(I_{q}^{0} f\right)(x)=f(x)$ and

$$
\begin{aligned}
\left(I_{q}^{\alpha} f\right)(x)=\frac{1}{\Gamma_{q}(\alpha)} \int_{0}^{x}(x-q t)^{(\alpha-1)} f(t) d_{q} t & \\
\alpha & >0, x \in[0, b] .
\end{aligned}
$$

Definition 3 (see [3]). The fractional $q$-derivative of the Caputo type of order $\alpha \geq 0$ is defined by

$$
\left({ }^{c} D_{q}^{\alpha} f\right)(x)=\left(I_{q}^{[\alpha]-\alpha} D_{q}^{[\alpha]} f\right)(x), \quad \alpha \geq 0,
$$

where $[\alpha]$ is the smallest integer greater than or equal to $\alpha$. If $f(x)=x^{\beta-1}, \beta>0$, then ${ }^{c} D_{q}^{\alpha} f(x)=\left(\Gamma_{q}(\beta) / \Gamma_{q}(\beta-\alpha)\right) x^{\beta-\alpha-1}$.

Lemma 4 (see $[2,3]$ ). Let $\alpha, \beta \geq 0$ and $f$ be a function defined on $[0, b]$. The following formulas hold:

(1) $\left(I_{q}^{\beta} I_{q}^{\alpha} f\right)(x)=\left(I_{q}^{\alpha+\beta} f\right)(x)$;

(2) $\left(D_{q}^{\alpha} I_{q}^{\alpha} f\right)(x)=f(x)$. 
Lemma 5 (see [3]). Let $\alpha \in \mathbb{R}^{+} \backslash \mathbb{N}$ and $a<x$. Then

$$
\left(I_{q}^{\alpha c} D_{q}^{\alpha} f\right)(x)=f(x)-\sum_{k=0}^{[\alpha]-1} \frac{\left(D_{q}^{k} f\right)(a)}{\Gamma_{q}(k+1)} x^{k}\left(\frac{a}{x}: q\right)_{k} .
$$

If $\alpha \geq m \geq \beta$, then ${ }^{c} D_{q}^{\beta} I_{q}^{\alpha} f(x)=I_{q}^{m-\beta} I_{q}^{\alpha-m} f(x)=I_{q}^{\alpha-\beta} f(x)$.

Lemma 6 (see [3]). For $\beta \in \mathbb{R}^{+}, \lambda \in(-1,+\infty)$ and $0 \leq a<$ $t \leq b$,

$$
I_{q}^{\beta}\left((t-a)^{(\lambda)}\right)=\frac{\Gamma_{q}(\lambda+1)}{\Gamma_{q}(\beta+\lambda+1)}(t-a)^{(\beta+\lambda)} .
$$

In particular, when $\lambda=0$ and $a=0$, using q-integration by part,

$$
\left(I_{q}^{\beta} 1\right)(t)=\frac{1}{\Gamma_{q(\beta)}} \int_{0}^{t}(t-q s)^{(\beta-1)} d_{q} s=\frac{1}{\Gamma_{q}(\beta+1)} t^{\beta} .
$$

Lemma 7 (see [32] (nonlinear alternative of Leray-Schauder type)). Let $X$ be a Banach space, $U$ be a bounded open subset of $X$ with $0 \in U$, and $P: \bar{U} \longrightarrow X$ be a completely continuous operator. Then, either there exists $x \in \partial U$ such that $x=\lambda P x$ for $\lambda \in(0,1)$ or there exists a fixed point $x^{*} \in \bar{U}$.

Let $P C(J, \mathbb{R})=\{u: u$ ia a map from $J$ into $\mathbb{R}$ such that $u(t)$ is continuous at $t \neq t_{k}$, left continuous at $t=t_{k}$ and its right limit at $t=t_{k}$ exists for $\left.k=1, \ldots, m\right\}$; then $P C(J, \mathbb{R})$ is a Banach space with the norm $\|u\|_{P C}=\sup \{|u(t)|: t \in J\}$.

Lemma 8. For $h \in P C(J, \mathbb{R})$, the solution of impulsive $B V P$,

$$
\begin{aligned}
{ }^{c} D_{q}^{\alpha} u(t) & =h(t), \quad t \in J^{\prime}, \\
\left.\Delta u\right|_{t=t_{k}} & =I_{k}\left(u\left(t_{k}^{-}\right)\right), \\
\left.\Delta D_{q} u\right|_{t=t_{k}} & =I_{k}^{*}\left(u\left(t_{k}^{-}\right)\right), \\
u(0) & =-u(1), \\
{ }^{c} D_{q}^{\beta} u(0) & =-{ }^{c} D_{q}^{\beta} u(1),
\end{aligned}
$$

is given by

$$
\begin{aligned}
& u(t)
\end{aligned}
$$

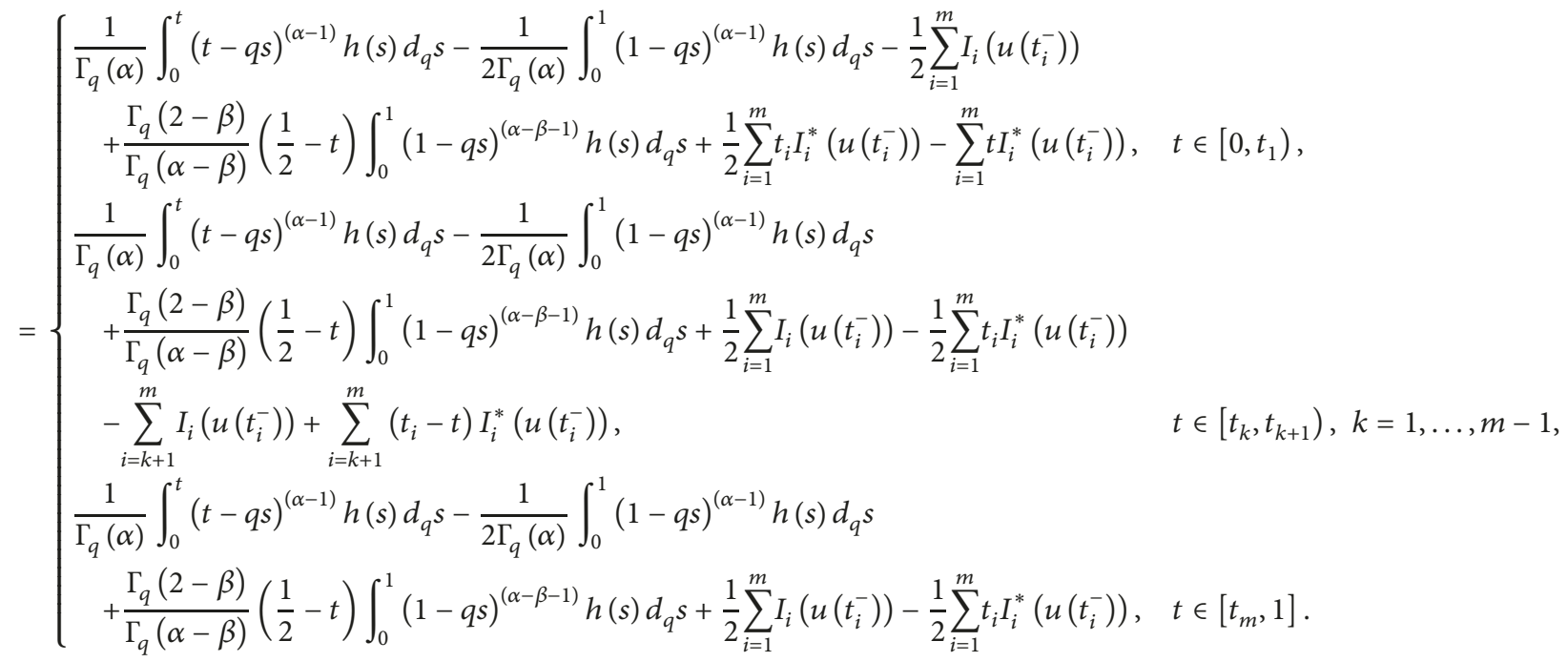

Proof. In view of Definitions 2 and 3 and Lemma 5, for $t \in$ $J_{k}=\left[t_{k}, t_{k+1}\right], k=0,1,2, \ldots, m$, we have

$$
\begin{aligned}
u(t) & =I_{q}^{\alpha} h(t)+d_{k}+e_{k} t \\
& =\frac{1}{\Gamma_{q}(\alpha)} \int_{0}^{t}(t-q s)^{(\alpha-1)} h(s) d_{q} s+d_{k}+e_{k} t,
\end{aligned}
$$

and

$$
\left(D_{q} u\right)(t)=\frac{1}{\Gamma_{q}(\alpha-1)} \int_{0}^{t}(t-q s)^{(\alpha-2)} h(s) d_{q} s+e_{k} .
$$

It follows from Definition 3, Lemma 5, and (18) that

$$
\begin{aligned}
{ }^{c} D_{q}^{\beta} u(t)= & \frac{1}{\Gamma_{q}(\alpha-\beta)} \int_{0}^{t}(t-q s)^{(\alpha-\beta-1)} h(s) d_{q} s \\
& +e_{k} \frac{t^{1-\beta}}{\Gamma_{q}(2-\beta)}, \quad t \in J_{k} .
\end{aligned}
$$

Applying ${ }^{c} D_{q}^{\beta} u(0)=-{ }^{c} D_{q}^{\beta} u(1)$ in (20), we obtain

$$
e_{m}=-\frac{\Gamma_{q}(2-\beta)}{\Gamma_{q}(\alpha-\beta)} \int_{0}^{1}(1-q s)^{(\alpha-\beta-1)} h(s) d_{q} s .
$$


Note boundary conditions $\left.\Delta u\right|_{t=t_{k}}=I_{k}\left(u\left(t_{k}^{-}\right)\right)$and $\left.\Delta D_{q} u\right|_{t=t_{k}}=I_{k}^{*}\left(u\left(t_{k}^{-}\right)\right)$; we get

$$
\begin{aligned}
d_{k}-d_{k-1}+\left(e_{k}-e_{k-1}\right) t_{k} & =I_{k}\left(u\left(t_{k}^{-}\right)\right), \\
e_{k}-e_{k-1} & =I_{k}^{*}\left(u\left(t_{k}^{-}\right)\right),
\end{aligned}
$$

$$
k=1,2, \ldots, m .
$$

Applying (21) and (22), we have

$$
\begin{aligned}
e_{k}= & e_{m}-\sum_{i=k+1}^{m} I_{i}^{*}\left(u\left(t_{i}^{-}\right)\right) \\
= & -\frac{\Gamma_{q}(2-\beta)}{\Gamma_{q}(\alpha-\beta)} \int_{0}^{1}(1-q s)^{(\alpha-\beta-1)} h(s) d_{q} s \\
& -\sum_{i=k+1}^{m} I_{i}^{*}\left(u\left(t_{i}^{-}\right)\right) .
\end{aligned}
$$

Thanks to $u(0)=-u(1)$, it is derived that

$$
d_{0}=-\frac{1}{\Gamma_{q}(\alpha)} \int_{0}^{1}(1-q s)^{(\alpha-1)} h(s) d_{q} s-d_{m}-e_{m}
$$

and

$$
\begin{aligned}
d_{m}+d_{0}= & -\frac{1}{\Gamma_{q}(\alpha)} \int_{0}^{1}(1-q s)^{(\alpha-1)} h(s) d_{q} s-e_{m} \\
= & -\frac{1}{\Gamma_{q}(\alpha)} \int_{0}^{1}(1-q s)^{(\alpha-1)} h(s) d_{q} s \\
& +\frac{\Gamma_{q}(2-\beta)}{\Gamma_{q}(\alpha-\beta)} \int_{0}^{1}(1-q s)^{(\alpha-\beta-1)} h(s) d_{q} s .
\end{aligned}
$$

By (22), we get

$$
d_{m}-d_{0}=\sum_{i=1}^{m} I_{i}\left(u\left(t_{i}^{-}\right)\right)-\sum_{i=1}^{m} t_{i} I_{i}^{*}\left(u\left(t_{i}^{-}\right)\right)
$$

Combining (25) and (26), we have

$$
\begin{aligned}
d_{m} & =\frac{1}{2}\left[-\frac{1}{\Gamma_{q}(\alpha)} \int_{0}^{1}(1-q s)^{(\alpha-1)} h(s) d_{q} s\right. \\
& +\frac{\Gamma_{q}(2-\beta)}{\Gamma_{q}(\alpha-\beta)} \int_{0}^{1}(1-q s)^{(\alpha-\beta-1)} h(s) d_{q} s \\
& \left.+\sum_{i=1}^{m} I_{i}\left(u\left(t_{i}^{-}\right)\right)-\sum_{i=1}^{m} t_{i} I_{i}^{*}\left(u\left(t_{i}^{-}\right)\right)\right],
\end{aligned}
$$

$$
\begin{aligned}
d_{k} & =d_{m}-\sum_{i=k+1}^{m} I_{i}\left(u\left(t_{i}^{-}\right)\right)+\sum_{i=k+1}^{m} t_{i} I_{i}^{*}\left(u\left(t_{i}^{-}\right)\right) \\
& =-\frac{1}{2 \Gamma_{q}(\alpha)} \int_{0}^{1}(1-q s)^{(\alpha-1)} h(s) d_{q} s+\frac{\Gamma_{q}(2-\beta)}{2 \Gamma_{q}(\alpha-\beta)} \\
& \cdot \int_{0}^{1}(1-q s)^{(\alpha-\beta-1)} h(s) d_{q} s+\frac{1}{2} \sum_{i=1}^{m} I_{i}\left(u\left(t_{i}^{-}\right)\right) \\
& -\frac{1}{2} \sum_{i=1}^{m} t_{i} I_{i}^{*}\left(u\left(t_{i}^{-}\right)\right)-\sum_{i=k+1}^{m} I_{i}\left(u\left(t_{i}^{-}\right)\right) \\
& +\sum_{i=k+1}^{m} t_{i} I_{i}^{*}\left(u\left(t_{i}^{-}\right)\right) .
\end{aligned}
$$

Therefore, for $t \in J_{k}, k=0,1,2, \ldots, m-1$,

$u(t)$

$$
\begin{aligned}
= & \frac{1}{\Gamma_{q}(\alpha)} \int_{0}^{t}(t-q s)^{(\alpha-1)} h(s) d_{q} s \\
& -\frac{1}{2 \Gamma_{q}(\alpha)} \int_{0}^{1}(1-q s)^{(\alpha-1)} h(s) d_{q} s \\
& +\frac{\Gamma_{q}(2-\beta)}{2 \Gamma_{q}(\alpha-\beta)} \int_{0}^{1}(1-q s)^{(\alpha-\beta-1)} h(s) d_{q} s \\
& +\frac{1}{2} \sum_{i=1}^{m} I_{i}\left(u\left(t_{i}^{-}\right)\right)-\frac{1}{2} \sum_{i=1}^{m} t_{i} I_{i}^{*}\left(u\left(t_{i}^{-}\right)\right) \\
& -\sum_{i=k+1}^{m} I_{i}\left(u\left(t_{i}^{-}\right)\right)+\sum_{i=k+1}^{m} t_{i} I_{i}^{*}\left(u\left(t_{i}^{-}\right)\right) \\
& -\frac{\Gamma_{q}(2-\beta)}{\Gamma_{q}(\alpha-\beta)} t \int_{0}^{1}(1-q s)^{(\alpha-\beta-1)} h(s) d_{q} s \\
& -t \sum_{i=k+1}^{m} I_{i}^{*}\left(u\left(t_{i}^{-}\right)\right)
\end{aligned}
$$$$
=\frac{1}{\Gamma_{q}(\alpha)} \int_{0}^{t}(t-q s)^{(\alpha-1)} h(s) d_{q} s
$$$$
-\frac{1}{2 \Gamma_{q}(\alpha)} \int_{0}^{1}(1-q s)^{(\alpha-1)} h(s) d_{q} s
$$$$
+\frac{\Gamma_{q}(2-\beta)}{\Gamma_{q}(\alpha-\beta)}\left(\frac{1}{2}-t\right) \int_{0}^{1}(1-q s)^{(\alpha-\beta-1)} h(s) d_{q} s
$$$$
+\frac{1}{2} \sum_{i=1}^{m} I_{i}\left(u\left(t_{i}^{-}\right)\right)-\frac{1}{2} \sum_{i=1}^{m} t_{i} I_{i}^{*}\left(u\left(t_{i}^{-}\right)\right)
$$$$
-\sum_{i=k+1}^{m} I_{i}\left(u\left(t_{i}^{-}\right)\right)+\sum_{i=k+1}^{m}\left(t_{i}-t\right) I_{i}^{*}\left(u\left(t_{i}^{-}\right)\right) \text {, }
$$ 
and, for $t \in J_{m}$,

$u(t)$

$$
\begin{aligned}
= & \frac{1}{\Gamma_{q}(\alpha)} \int_{0}^{t}(t-q s)^{(\alpha-1)} h(s) d_{q} s \\
& -\frac{1}{2 \Gamma_{q}(\alpha)} \int_{0}^{1}(1-q s)^{(\alpha-1)} h(s) d_{q} s \\
& +\frac{\Gamma_{q}(2-\beta)}{\Gamma_{q}(\alpha-\beta)}\left(\frac{1}{2}-t\right) \int_{0}^{1}(1-q s)^{(\alpha-\beta-1)} h(s) d_{q} s \\
& +\frac{1}{2} \sum_{i=1}^{m} I_{i}\left(u\left(t_{i}^{-}\right)\right)-\frac{1}{2} \sum_{i=1}^{m} t_{i} I_{i}^{*}\left(u\left(t_{i}^{-}\right)\right) .
\end{aligned}
$$

\section{Main Results}

Define an operator $A: P C(J, \mathbb{R}) \longrightarrow P C(J, \mathbb{R})$ by

$$
\begin{aligned}
& A u(t)=\frac{1}{\Gamma_{q}(\alpha)} \\
& \cdot \int_{0}^{t}(t-q s)^{(\alpha-1)} f(s, u(s), T u(s), S u(s)) d_{q} \\
& \quad-\frac{1}{2 \Gamma_{q}(\alpha)} \\
& \cdot \int_{0}^{1}(1-q s)^{(\alpha-1)} f(s, u(s), T u(s), S u(s)) d_{q} s \\
& +\frac{\Gamma_{q}(2-\beta)}{\Gamma_{q}(\alpha-\beta)}\left(\frac{1}{2}-t\right) \\
& \quad \cdot \int_{0}^{1}(1-q s)^{(\alpha-\beta-1)} f(s, u(s), T u(s), S u(s)) d_{q} s \\
& +\frac{1}{2} \sum_{i=1}^{m} I_{i}\left(u\left(t_{i}^{-}\right)\right)-\frac{1}{2} \sum_{i=1}^{m} t_{i} I_{i}^{*}\left(u\left(t_{i}^{-}\right)\right) \\
& \quad-\sum_{i=k+1}^{m} I_{i}\left(u\left(t_{i}^{-}\right)\right)+\sum_{i=k+1}^{m}\left(t_{i}-t\right) I_{i}^{*}\left(u\left(t_{i}^{-}\right)\right) \\
& \quad t \in J_{k}, k=0,1, \ldots, m .
\end{aligned}
$$

Theorem 9. Assume that

$\left(H_{1}\right)$ There exist nonnegative functions $L_{j}(t) \in C(J)(j=$ $1,2,3)$ such that

$$
\begin{aligned}
& \left|f\left(t, u_{1}, v_{1}, w_{1}\right)-f\left(t, u_{2}, v_{2}, w_{2}\right)\right| \\
& \quad \leq L_{1}(t)\left|u_{1}-u_{2}\right|+L_{2}(t)\left|v_{1}-v_{2}\right| \\
& \quad+L_{3}(t)\left|w_{1}-w_{2}\right|
\end{aligned}
$$

for $t \in J, u_{i}, v_{i}, \omega_{i} \in \mathbb{R}, i=1,2$.
$\left(\mathrm{H}_{2}\right)$ There exist positive numbers $N$ and $N^{*}$ such that

$$
\begin{gathered}
\left|I_{k}(u)-I_{k}(v)\right| \leq N|u-v|, \\
\left|I_{k}^{*}(u)-I_{k}^{*}(v)\right| \leq N^{*}|u-v|,
\end{gathered}
$$

$\left(H_{3}\right)$

$$
\begin{aligned}
\chi= & \frac{3\left(\overline{L_{1}}+\overline{L_{2}} k_{0}+\overline{L_{3}} h_{0}\right)}{2 \Gamma_{q}(\alpha+1)} \\
& +\frac{\Gamma_{q}(2-\beta)\left(\overline{L_{1}}+\overline{L_{2}} k_{0}+\overline{L_{3}} h_{0}\right)}{2 \Gamma_{q}(\alpha-\beta+1)}+\frac{3}{2} m N \\
& +\frac{5}{2} m N^{*}<1,
\end{aligned}
$$

where $\overline{L_{i}}=\max \left\{L_{i}(t): t \in J\right\}, \quad i=1,2,3, k_{0}=\max \{|k(t, s)|:$ $(t, s) \in D\}, h_{0}=\max \left\{|h(t, s)|:(t, s) \in D_{0}\right\}$.

Then BVP (1) has a unique solution.

Proof. For $u, v \in P C(J, \mathbb{R})$ and $t \in J$, we have

$$
\begin{aligned}
& |(A u)(t)-(A v)(t)| \\
& \leq \int_{0}^{t} \frac{(t-q s)^{(\alpha-1)}}{\Gamma_{q}(\alpha)} \mid f(s, u(s), T u(s), S u(s)) \\
& -f(s, v(s), T v(s), S v(s)) \mid d_{q} s+\frac{1}{2} \\
& \cdot \int_{0}^{1} \frac{(1-q s)^{(\alpha-1)}}{\Gamma_{q}(\alpha)} \mid f(s, u(s), T u(s), S u(s)) \\
& -f(s, v(s), T v(s), S v(s)) \mid d_{q} s \\
& +\frac{\Gamma_{q}(2-\beta)}{2 \Gamma_{q}(\alpha-\beta)} \int_{0}^{1}(1-q s)^{(\alpha-\beta-1)} \\
& \cdot \mid f(s, u(s), T u(s), S u(s)) \\
& -f(s, v(s), T v(s), S v(s)) \mid d_{q} s+\frac{1}{2} \\
& \cdot \sum_{i=1}^{m}\left|I\left(u\left(t_{i}^{-}\right)\right)-I_{i}\left(v\left(t_{i}^{-}\right)\right)\right|_{i}+\frac{1}{2} \sum_{i=1}^{m} t_{i} \mid I_{i}^{*}\left(u\left(t_{i}^{-}\right)\right) \\
& -I_{i}^{*}\left(v\left(t_{i}^{-}\right)\right)\left|+\sum_{i=1}^{m}\right| I_{i}\left(u\left(t_{i}^{-}\right)\right)-I_{i}\left(v\left(t_{i}^{-}\right)\right) \mid \\
& +\sum_{i=1}^{m} t_{i}\left|I_{i}^{*}\left(u\left(t_{i}^{-}\right)\right)-I_{i}^{*}\left(v\left(t_{i}^{-}\right)\right)\right|+t \sum_{i=1}^{m} \mid I_{i}^{*}\left(u\left(t_{i}^{-}\right)\right) \\
& -I_{i}^{*}\left(v\left(t_{i}^{-}\right)\right) \mid \leq \frac{1}{\Gamma_{q}(\alpha)} \int_{0}^{t}(t-q s)^{(\alpha-1)} \\
& \cdot\left(L_{1}(s)|u(s)-v(s)|\right.
\end{aligned}
$$




$$
\begin{aligned}
& +L_{2}(s)\left|\int_{0}^{s} k(s, \tau)(u(\tau)-v(\tau)) d_{q} \tau\right| \\
& \left.+L_{3}(s)\left|\int_{0}^{1} h(s, \tau)(u(\tau)-v(\tau)) d_{q} \tau\right|\right) d_{q} s \\
& +\frac{1}{2 \Gamma_{q}(\alpha)} \int_{0}^{1}(1-q s)^{(\alpha-1)}\left(L_{1}(s)|u(s)-v(s)|\right. \\
& +L_{2}(s)\left|\int_{0}^{s} k(s, \tau)(u(\tau)-v(\tau)) d_{q} \tau\right| \\
& \left.+L_{3}(s)\left|\int_{0}^{1} h(s, \tau)(u(\tau)-v(\tau)) d_{q} \tau\right|\right) d_{q} s \\
& +\frac{\Gamma_{q}(2-\beta)}{2 \Gamma_{q}(\alpha-\beta)} \int_{0}^{1}(1-q s)^{(\alpha-\beta-1)} \\
& \cdot\left(L_{1}(s)|u(s)-v(s)|\right. \\
& +L_{2}(s)\left|\int_{0}^{s} k(s, \tau)(u(\tau)-v(\tau)) d_{q} \tau\right| \\
& \left.+L_{3}(s)\left|\int_{0}^{1} h(s, \tau)(u(\tau)-v(\tau)) d_{q} \tau\right|\right) d_{q} s \\
& +\frac{3}{2} \sum_{i=1}^{m}\left|I_{i}\left(u\left(t_{i}^{+}\right)\right)-I_{i}\left(v\left(t_{i}^{-}\right)\right)\right|+\sum_{i=1}^{m}\left(\frac{3}{2} t_{i}+t\right) \\
& \cdot\left|I_{i}^{*}\left(u\left(t_{i}^{+}\right)\right)-I_{i}^{*}\left(v\left(t_{i}^{-}\right)\right)\right| \leq \frac{1}{\Gamma_{q}(\alpha)} \int_{0}^{t}(t \\
& -q s)^{(\alpha-1)}\left(\overline{L_{1}}\|u-v\|_{P C}+\overline{L_{2}} k_{0}\|u-v\|_{P C}\right. \\
& \left.+\overline{L_{3}} h_{0}\|u-v\|_{P C}\right) d_{q} s+\frac{1}{2 \Gamma_{q}(\alpha)} \int_{0}^{1}(1 \\
& -q s)^{(\alpha-1)}\left(\overline{L_{1}}\|u-v\|_{P C}+\overline{L_{2}} k_{0}\|u-v\|_{P C}\right. \\
& \left.+\overline{L_{3}} h_{0}\|u-v\|_{P C}\right) d_{q} s+\frac{\Gamma_{q}(2-\beta)}{2 \Gamma_{q}(\alpha-\beta)} \int_{0}^{1}(1 \\
& -q s)^{(\alpha-\beta-1)}\left(\overline{L_{1}}\|u-v\|_{P C}+\overline{L_{2}} k_{0}\|u-v\|_{P C}\right. \\
& \left.+\overline{L_{3}} h_{0}\|u-v\|_{P C}\right) d_{q} s+\frac{3}{2} m N\|u-v\|_{P C} \\
& +\frac{5}{2} m N^{*}\|u-v\|_{P C} \leq\left(\overline{L_{1}}+\overline{L_{2}} k_{0}+\overline{L_{3}} h_{0}\right) \\
& \cdot\left(\frac{1}{\Gamma_{q}(\alpha+1)}+\frac{1}{2 \Gamma_{q}(\alpha+1)}+\frac{\Gamma_{q}(2-\beta)}{2 \Gamma_{q}(\alpha-\beta+1)}\right) \| u \\
& -v\left\|_{P C}+\left(\frac{3}{2} m N+\frac{5}{2} m N^{*}\right)\right\| u-v \|_{P C} \\
& =\left\{\frac{3\left(\overline{L_{1}}+\overline{L_{2}} k_{0}+\overline{L_{3}} h_{0}\right)}{2 \Gamma_{q}(\alpha+1)}\right.
\end{aligned}
$$

$$
\begin{aligned}
& +\frac{\Gamma_{q}(2-\beta)\left(\overline{L_{1}}+\overline{L_{2}} k_{0}+\overline{L_{3}} h_{0}\right)}{2 \Gamma_{q}(\alpha-\beta+1)}+\frac{3}{2} m N+\frac{5}{2} \\
& \left.\cdot m N^{*}\right\}\|u-v\|_{P C}=\chi\|u-v\|_{P C},
\end{aligned}
$$

and then $\|A u-A v\|_{P C} \leq \chi\|u-v\|_{P C}$, and hence $A$ is a contraction operator. It follows from Banach contraction mapping principle that BVP (1) has a unique solution.

Theorem 10. Assume the following:

$\left(\mathrm{H}_{4}\right)$ There exist continuous and nondecreasing function $g$ : $[0,+\infty) \longrightarrow(0,+\infty)$ and $a(t) \in C[0,1]$ such that

$$
\begin{aligned}
|f(t, u, v, w)| \leq a(t) g(\max \{|u|,|v|,|w|\}), & \\
& t \in[0,1], u, v, w \in \mathbb{R} .
\end{aligned}
$$

$\left(\mathrm{H}_{5}\right)$ There exist continuous and nondecreasing functions $\varphi, \psi:[0,+\infty) \longrightarrow(0,+\infty)$ such that

$$
\begin{aligned}
& \left|I_{k}(u)\right| \leq \varphi(|u|), \\
& \left|I_{k}^{*}(u)\right| \leq \psi(|u|),
\end{aligned}
$$

$u \in \mathbb{R}, k=1, \ldots, m$.

$\left(H_{6}\right)$ There exists constant $M>0$ such that

$$
\begin{aligned}
M> & a^{\prime} g\left(\max \left\{M, k_{0} M, h_{0} M\right\}\right) \\
& \cdot\left(\frac{3}{2 \Gamma_{q}(\alpha+1)}+\frac{\Gamma_{q}(2-\beta)}{2 \Gamma_{q}(\alpha-\beta+1)}\right)+\frac{3 m}{2} \varphi(M) \\
& +\frac{5}{2} m \psi(M),
\end{aligned}
$$

where $a^{\prime}=\max \{a(t): t \in[0,1]\}$.

Then BVP (1) has at least one solution.

Proof. The continuity of $f, I_{k}, I_{k}^{*}$ implies that operator $A$ : $P C(J, \mathbb{R}) \longrightarrow P C(J, \mathbb{R})$ is continuous. Let $B \subset P C(J, \mathbb{R})$ be bounded; then there exist positive constants $P_{1}, P_{2}$, and $P_{3}$ such that $|f(t, u(t), T u(t), S u(t))| \leq P_{1},\left|I_{i}\left(u\left(t_{i}^{-}\right)\right)\right| \leq P_{2}$, and $\left|I_{i}^{*}\left(u\left(t_{i}^{-}\right)\right)\right| \leq P_{3}$ for all $t \in J, u \in B, i=1,2, \ldots, m$. Thus, we have

$$
\begin{aligned}
|(A u)(t)| \leq & \int_{0}^{t} \frac{(t-q s)^{(\alpha-1)}}{\Gamma_{q}(\alpha)} P_{1} d_{q} s \\
& +\frac{1}{2} \int_{0}^{1} \frac{(1-q s)^{(\alpha-1)}}{\Gamma_{q}(\alpha)} P_{1} d_{q} s
\end{aligned}
$$




$$
\begin{aligned}
& +\frac{1}{2} \Gamma_{q}(2-\beta) \int_{0}^{1} \frac{(1-q s)^{(\alpha-\beta-1)}}{\Gamma_{q}(\alpha-\beta)} P_{1} d_{q} s \\
& +\frac{m}{2} P_{2}+\frac{P_{3}}{2} \sum_{i=1}^{m} t_{i}+m P_{2}+P_{3} \sum_{i=1}^{m} t_{i}+m P_{3} \\
& \leq \frac{3 P_{1}}{2 \Gamma_{q}(\alpha+1)}+\frac{\Gamma_{q}(2-\beta)}{2 \Gamma_{q}(\alpha-\beta+1)} P_{1}+\frac{3 m}{2} P_{2} \\
& \quad+\frac{5}{2} m P_{3} .
\end{aligned}
$$

Consequently, operator $A$ is uniformly bounded on $B$. have

On the other hand, for $t_{k} \leq \tau_{1}<\tau_{2} \leq t_{k+1}, u \in B$, we

$$
\begin{aligned}
& \left|(A u)\left(\tau_{2}\right)-(A u)\left(\tau_{1}\right)\right|=\mid \frac{1}{\Gamma_{q}(\alpha)} \int_{0}^{\tau_{2}}\left(\tau_{2}-q s\right)^{(\alpha-1)} \\
& \cdot f(s, u(s), T u(s), S u(s)) d_{q} s-\frac{1}{\Gamma_{q}(\alpha)} \\
& \cdot \int_{0}^{\tau_{1}}\left(\tau_{1}-q s\right)^{(\alpha-1)} \\
& \cdot f(s, u(s), T u(s), S u(s)) d_{q} s \\
& +\frac{\Gamma_{q}(2-\beta)}{\Gamma_{q}(\alpha-\beta)}\left(\tau_{1}-\tau_{2}\right) \int_{0}^{1}(1-q s)^{(\alpha-\beta-1)} \\
& \cdot f(s, u(s), T u(s), S u(s)) d_{q} s \\
& +\sum_{i=k+1}^{m} I_{i}^{*}\left(u\left(t_{i}^{-}\right)\right)\left(\tau_{1}-\tau_{2}\right) \mid \leq \frac{1}{\Gamma_{q}(\alpha)} \\
& \cdot \int_{0}^{\tau_{1}}\left|\left(\tau_{2}-q s\right)^{(\alpha-1)}-\left(\tau_{1}-q s\right)^{(\alpha-1)}\right| \\
& \cdot|f(s, u(s), T u(s), S u(s))| d_{q} s+\frac{1}{\Gamma_{q}(\alpha)} \\
& \cdot \int_{\tau_{1}}^{\tau_{2}}\left|\left(\tau_{2}-q s\right)^{(\alpha-1)} f(s, u(s), T u(s), S u(s))\right| d_{q} s \\
& +\frac{\Gamma_{q}(2-\beta)}{\Gamma_{q}(\alpha-\beta)} P_{1}\left|\tau_{2}-\tau_{1}\right| \int_{0}^{1}(1-q s)^{(\alpha-\beta-1)} d_{q} s \\
& +m P_{3}\left|\tau_{2}-\tau_{1}\right| \leq P_{1} \frac{\tau_{2}^{\alpha}-\tau_{1}^{\alpha}}{\Gamma_{q}(\alpha+1)}+P_{1} \\
& \frac{\Gamma_{q}(2-\beta)}{\Gamma_{q}(\alpha-\beta+1)}\left|\tau_{2}-\tau_{1}\right|+m P_{3}\left|\tau_{2}-\tau_{1}\right| \text {, }
\end{aligned}
$$

which tends to zero as $\tau_{2} \longrightarrow \tau_{1}$; then $A$ is equicontinuous on $J_{k}$. Hence by $P C$-type Arzela-Ascoli Theorem ([33]), operator $A: P C(J, \mathbb{R}) \longrightarrow P C(J, \mathbb{R})$ is completely continuous.
Let $u \in P C(J, \mathbb{R})$ be such that $u(t)=\lambda(A u)(t)$ for some $\lambda \in(0,1)$; then

$$
\begin{aligned}
& |u(t)|=|\lambda(A u)(t)| \leq|(A u)(t)| \\
& \quad \leq \int_{0}^{t} \frac{(t-q s)^{(\alpha-1)}}{\Gamma_{q}(\alpha)} a^{\prime} g\left(\operatorname { m a x } \left\{\|u\|_{P C}, k_{0}\|u\|_{P C},\right.\right. \\
& \left.\left.h_{0}\|u\|_{P C}\right\}\right) d_{q} s+\frac{1}{2} \\
& \cdot \int_{0}^{1} \frac{(1-q s)^{(\alpha-1)}}{\Gamma_{q}(\alpha)} a^{\prime} g\left(\operatorname { m a x } \left\{\|u\|_{P C}, k_{0}\|u\|_{P C},\right.\right.
\end{aligned}
$$

$\left.\left.h_{0}\|u\|_{P C}\right\}\right) d_{q} s+\frac{1}{2} \Gamma_{q}(2-\beta)$

$\cdot \int_{0}^{1} \frac{(1-q s)^{(\alpha-\beta-1)}}{\Gamma_{q}(\alpha-\beta)} a^{\prime} g\left(\max \left\{\|u\|_{P C}, k_{0}\|u\|_{P C}\right.\right.$,

$\left.\left.h_{0}\|u\|_{P C}\right\}\right) d_{q} s+\frac{1}{2} m \varphi\left(\|u\|_{P C}\right)+\frac{1}{2}$

$\cdot \psi\left(\|u\|_{P C}\right) \sum_{i=1}^{m} t_{i}+m \varphi\left(\|u\|_{P C}\right)+\psi\left(\|u\|_{P C}\right) \sum_{i=1}^{m} t_{i}$

$+m \psi\left(\|u\|_{P C}\right) \leq a^{\prime} g\left(\max \left\{\|u\|_{P C}, k_{0}\|u\|_{P C}\right.\right.$,

$\left.\left.h_{0}\|u\|_{P C}\right\}\right)\left(\frac{3}{2 \Gamma_{q}(\alpha+1)}+\frac{\Gamma_{q}(2-\beta)}{2 \Gamma_{q}(\alpha-\beta+1)}\right)+\frac{3 m}{2}$

$\cdot \varphi\left(\|u\|_{P C}\right)+\frac{5}{2} m \psi\left(\|u\|_{P C}\right)$

and hence

$\|u\|_{P C} \leq a^{\prime} g\left(\max \left\{\|u\|_{P C}, k_{0}\|u\|_{P C}, h_{0}\|u\|_{P C}\right\}\right)$

$$
\begin{aligned}
& \cdot\left(\frac{3}{2 \Gamma_{q}(\alpha+1)}+\frac{\Gamma_{q}(2-\beta)}{2 \Gamma_{q}(\alpha-\beta+1)}\right)+\frac{3 m}{2} \\
& \cdot \varphi\left(\|u\|_{P C}\right)+\frac{5}{2} m \psi\left(\|u\|_{P C}\right) .
\end{aligned}
$$

Let $U=\{u \in P C(J, \mathbb{R}):\|u\|<M\}$; then operator $A: \bar{U} \longrightarrow$ $P C(J, \mathbb{R})$ is completely continuous. By $\left(H_{6}\right)$, one has $u \neq \lambda A u$ for any $\lambda \in(0,1)$ and $u \in \partial U$. By Lemma 7, BVP (1) has at least one solution.

\section{Examples}

Example 1. Consider the BVP

$$
\begin{aligned}
{ }^{c} D_{1 / 2}^{3 / 2} u(t)= & \frac{u(t)+1}{100}+\frac{1}{50+t^{2}} \int_{0}^{t} \frac{u(s)}{e^{(t+s)}} d_{q} s \\
& +\frac{e^{-t}}{80} \int_{0}^{1} \frac{u(s)}{2+t+s} d_{q} s, \\
& t \in[0,1] \backslash\left\{\frac{1}{2}\right\},
\end{aligned}
$$




$$
\begin{aligned}
\left.\Delta u\right|_{t=1 / 2} & =\frac{|u(1 / 2)|}{10+|u(1 / 2)|}, \\
\left.\Delta D_{1 / 2} u\right|_{t=1 / 2} & =\frac{|u(1 / 2)|}{20+|u(1 / 2)|} \\
u(0) & =-u(1), \\
{ }^{c} D_{1 / 2}^{1 / 4} u(0) & =-{ }^{c} D_{1 / 2}^{1 / 4} u(1) .
\end{aligned}
$$

Let

$$
\begin{gathered}
f(t, u, v, w)=\frac{u+1}{100}+\frac{1}{50+t^{2}} v+\frac{e^{-t}}{80} w, \\
(T u)(t)=\int_{0}^{t} e^{-(t+s)} u(s) d_{q} s, \\
(S u)(t)=\int_{0}^{1} \frac{u(s)}{2+t+s} d_{q} s .
\end{gathered}
$$

By direct computation, $k_{0}=\max \left\{e^{-(t+s)}: 0 \leq s \leq t \leq 1\right\}=$ $1, h_{0}=\max \{1 /(2+t+s): 0 \leq s, t \leq 1\}=1 / 2$. For any $u_{1}, u_{2}, v_{1}, v_{2}, \omega_{1}, \omega_{2} \in \mathbb{R}$ and $t \in J$, we have

$$
\begin{aligned}
& \left|f\left(t, u_{1}, v_{1}, w_{1}\right)-f\left(t, u_{2}, v_{2}, w_{2}\right)\right| \\
& \quad \leq \frac{1}{100}\left|u_{1}-u_{2}\right|+\frac{1}{50}\left|v_{1}-v_{2}\right|+\frac{1}{80}\left|w_{1}-w_{2}\right|, \\
& \left|I_{k}(u)-I_{k}(v)\right| \leq \frac{1}{10}|u-v| \\
& \left|I_{k}^{*}(u)-I_{k}^{*}(v)\right| \leq \frac{1}{20}|u-v| .
\end{aligned}
$$

Let $L_{1}(t)=1 / 100, L_{2}(t)=1 / 50, L_{3}(t)=1 / 80, N=1 / 10$, and $N^{*}=1 / 20$; then

$$
\begin{aligned}
\chi= & \frac{3\left(\overline{L_{1}}+\overline{L_{2}} k_{0}+\overline{L_{3}} h_{0}\right)}{2 \Gamma_{1 / 2}(5 / 2)} \\
& +\frac{\Gamma_{1 / 2}(7 / 4)\left(\overline{L_{1}}+\overline{L_{2}} k_{0}+\overline{L_{3}} h_{0}\right)}{2 \Gamma_{1 / 2}(9 / 4)}+\frac{3}{2} N+\frac{5}{2} N^{*} \\
\approx & 0.307<1 .
\end{aligned}
$$

Then, $\left(H_{1}\right)-\left(H_{3}\right)$ hold. It follows from Theorem 9 that BVP (42) has a unique solution.

Example 2. Consider the BVP

$$
\begin{aligned}
& { }^{c} D_{q}^{3 / 2} u(t)=\frac{t^{2}}{60}\left(\sin u(t)+\int_{0}^{t} \cos (u(s)) d_{q} s\right. \\
& \left.+\int_{0}^{1} \frac{1}{(u(s))^{2}+t^{2}+1} d_{q} s\right), \quad t \in[0,1] \backslash\left\{\frac{1}{2}\right\}, \\
& \left.\Delta u\right|_{t=1 / 2}=\frac{|u(1 / 2)|}{20+|u(1 / 2)|},
\end{aligned}
$$

$$
\begin{aligned}
& \left.\Delta D_{q} u\right|_{t=1 / 2}=\frac{|u(1 / 2)|}{30+|u(1 / 2)|}, \\
& u(0)=-u(1), \\
& { }^{c} D_{q}^{1 / 4} u(0)=-{ }^{c} D_{q}^{1 / 4} u(1) .
\end{aligned}
$$

Let

$$
\begin{aligned}
f(t, u, v, w) & =\frac{t^{2}}{60}(\sin u+v+w), \\
(T u)(t) & =\int_{0}^{t} \cos (u(s)) d_{q} s \\
(S u)(t) & =\int_{0}^{1} \frac{d_{q} s}{(u(s))^{2}+t^{2}+1}
\end{aligned}
$$

then

$$
\begin{aligned}
& |f(t, u(t), T u(t), S u(t))| \leq \frac{1}{60}(1+t+1) \\
& \quad \leq 6(t+1) .
\end{aligned}
$$

Let $g(r)=6, a(t)=t+1$; then $a^{\prime}=\max \{t+1: t \in[0,1]\}=2$. Choose $\varphi(u)=1$ and $\psi(u)=1$; we have

$$
\begin{aligned}
2 \times & 6 \times\left(\frac{3}{2 \Gamma_{1 / 2}(5 / 2)}+\frac{\Gamma_{1 / 2}(7 / 4)}{2 \Gamma_{1 / 2}(9 / 4)}\right)+\frac{3}{2}+\frac{5}{2} \\
& \approx 19.87
\end{aligned}
$$

Let $M=20$; then condition $\left(H_{6}\right)$ holds. Therefore, by Theorem 10, BVP (46) has at least one solution.

\section{Data Availability}

No data were used to support this study.

\section{Conflicts of Interest}

The authors declare that there are no conflicts of interest.

\section{Authors' Contributions}

The authors contributed equally to this paper. All authors read and approved the final manuscript.

\section{Acknowledgments}

Supported financially by the National Natural Science Foundation of China (11501318, 11871302), the China Postdoctoral Science Foundation (2017M612230), the Natural Science Foundation of Shandong Province of China (ZR2017MA036), and the International Cooperation Program of Key Professors by Qufu Normal University. 


\section{References}

[1] W. A. Al-Salam, "Some fractional $q$-integrals and $q$-derivatives," Proceedings of the Edinburgh Mathematical Society, vol. 15, no. 2, pp. 135-140, 1967.

[2] R. Agarwal, "Certain fractional $q$-integrals and $q$-derivatives," Mathematical Proceedings of the Cambridge Philosophical Society, vol. 66, no. 2, pp. 365-370, 1969.

[3] P. M. Rajković, S. D. Marinković, and M. S. Stanković, "Fractional integrals and derivatives in q-calculus," Applicable Analysis and Discrete Mathematics, vol. 1, no. 1, pp. 311-323, 2007.

[4] D. Baleanu, K. Diethelm, E. Scalas, and J. J. Trujillo, Fractional Calculus Models and Numerical Methods, vol. 3 of Series on Complexity, Nonlinearity and Chaos, World Scientific, Boston, MA, USA, 2012.

[5] A. M. Samoilenko and N. A. Perestyuk, Impulsive Differential Equations, World Scientific, Singapore, 1995.

[6] V. Lakshmikantham, D. D. Bainov, and P. S. Simeonov, Theory of Impulsive Differential Equations, World Scientific, Singapore, 1989.

[7] I. Rachunkova and J. Tomecek, "Existence principle for BVPS with state-dependent impulses," Topological Methods in Nonlinear Analysis, vol. 44, pp. 349-368, 2014.

[8] X. Zhang, "Exact interval of parameter and two infinite families of positive solutions for a $n$th order impulsive singular equation," Journal of Computational and Applied Mathematics, vol. 330, pp. 896-908, 2018.

[9] X. Zhang and M. Feng, "Nontrivial convex solutions on a parameter of impulsive differential equation with MongeAmpere operator," Boundary Value Problems, vol. 2017, no. 172, 2017.

[10] X. Zhang, L. Liu, Y. Wu, and Y. Cui, "Existence of infinitely solutions for a modified nonlinear Schrodinger equation via dual approach," Electronic Journal of Differential Equations, no. 147, p. 15, 2018.

[11] M. Feng, B. Du, and W. Ge, "Impulsive boundary value problems with integral boundary conditions and one-dimensional p-Laplacian," Nonlinear Analysis: Theory, Methods \& Applications, vol. 70, no. 9, pp. 3119-3126, 2009.

[12] J. Henderson and R. Luca, "Positive solutions for an impulsive second-order nonlinear boundary value problem," Mediterranean Journal of Mathematics, vol. 14, no. 93, 2017.

[13] X. Zhang, L. Liu, Y. Wu, and Y. Cui, "Entire blow-up solutions for a quasilinear $p$-Laplacian Schrödinger equation with a nonsquare diffusion term," Applied Mathematics Letters, vol. 74, pp. 85-93, 2017.

[14] G. Bonanno, B. Di Bella, and J. Henderson, "Infinitely many solutions for a boundary value problem with impulsive effects," Boundary Value Problems, vol. 2013, no. 278, 2013.

[15] X. Hao, M. Zuo, and L. Liu, "Multiple positive solutions for a system of impulsive integral boundary value problems with sign-changing nonlinearities," Applied Mathematics Letters, vol. 82, pp. 24-31, 2018.

[16] X. Hao and L. Liu, "Mild solution of semilinear impulsive integro-differential evolution equation in Banach spaces," Mathematical Methods in the Applied Sciences, vol. 40, no. 13, pp. 4832-4841, 2017.

[17] F. Yan, M. Zuo, and X. Hao, "Positive solution for a fractional singular boundary value problem with $p$-Laplacian operator," Boundary Value Problems, no. 51, 2018.
[18] X. Hao, L. Liu, and Y. Wu, "Positive solutions for second order impulsive differential equations with integral boundary conditions," Communications in Nonlinear Science and Numerical Simulation, vol. 16, no. 1, pp. 101-111, 2011.

[19] X. Zhang, Y. Wu, and Y. Cui, "Existence and nonexistence of blow-up solutions for a Schrödinger equation involving a nonlinear operator," Applied Mathematics Letters, vol. 82, pp. 85-91, 2018.

[20] X. Zhang, L. Liu, Y. Wu, and Y. Cui, “The existence and nonexistence of entire large solutions for a quasilinear Schrödinger elliptic system by dual approach," Journal of Mathematical Analysis and Applications, vol. 464, no. 2, pp. 1089-1106, 2018.

[21] J. Liu and Z. Zhao, "Multiple solutions for impulsive problems with non-autonomous perturbations," Applied Mathematics Letters, vol. 64, pp. 143-149, 2017.

[22] X. Li, F. Chen, and X. Li, "Generalized anti-periodic boundary value problems of impulsive fractional differential equations," Communications in Nonlinear Science and Numerical Simulation, vol. 18, no. 1, pp. 28-41, 2013.

[23] J. Wang and Z. Lin, "On the impulsive fractional anti-periodic BVP modelling with constant coefficients," Journal of Applied Mathematics and Computing, vol. 46, no. 1-2, pp. 107-121, 2014.

[24] L. H. Zhang and G. Wang, "Existence of solutions for nonlinear fractional differential equations with impulses and antiperiodic boundary conditions," Electronic Journal of Qualitative Theory of Differential Equations, vol. 2011, no. 7, pp. 1-11, 2011.

[25] A. Chen and Y. Chen, "Existence of solutions to anti-periodic boundary value problem for nonlinear fractional differential equations with impulses," Advances in Difference Equations, Article ID 915689, 17 pages, 2011.

[26] X. Li, Z. Han, and S. Sun, "Anti-periodic boundary value problems for fractional q-difference equations," Journal of Applied Mathematics and Computing, vol. 50, no. 1-2, pp. 243-257, 2016.

[27] X. Hao and H. Wang, "Positive solutions of semipositone singular fractional differential systems with a parameter and integral boundary conditions," Open Mathematics, vol. 16, pp. 581-596, 2018.

[28] B. Ahmad, J. Tariboon, S. K. Ntouyas, H. H. Alsulami, and S. Monaquel, "Existence results for impulsive fractional $q$ difference equations with anti-periodic boundary conditions," Boundary Value Problems, no. 16, 2016.

[29] Z. Bai, X. Dong, and C. Yin, "Existence results for impulsive nonlinear fractional differential equation with mixed boundary conditions," Boundary Value Problems, no. 63, 2016.

[30] X. Fu and X. Bao, "Some existence results for nonlinear fractional differential equations with impulsive and fractional integral boundary conditions," Advances in Difference Equations, no. 129, 2014.

[31] M. H. Annaby and Z. S. Mansour, q-Fractional Calculus and Equations, vol. 2056 of Lecture Notes in Mathematics, Springer, Berlin, Germany, 2012.

[32] K. Deimling, Nonlinear Functional Analysis, Springer, Berlin , Germany, 1985.

[33] W. Wei, X. Xiang, and Y. Peng, "Nonlinear impulsive integrodifferential equations of mixed type and optimal controls," Optimization. A Journal of Mathematical Programming and Operations Research, vol. 55, no. 1-2, pp. 141-156, 2006. 


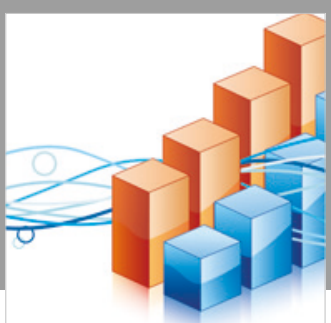

Advances in

Operations Research

\section{-n-m}
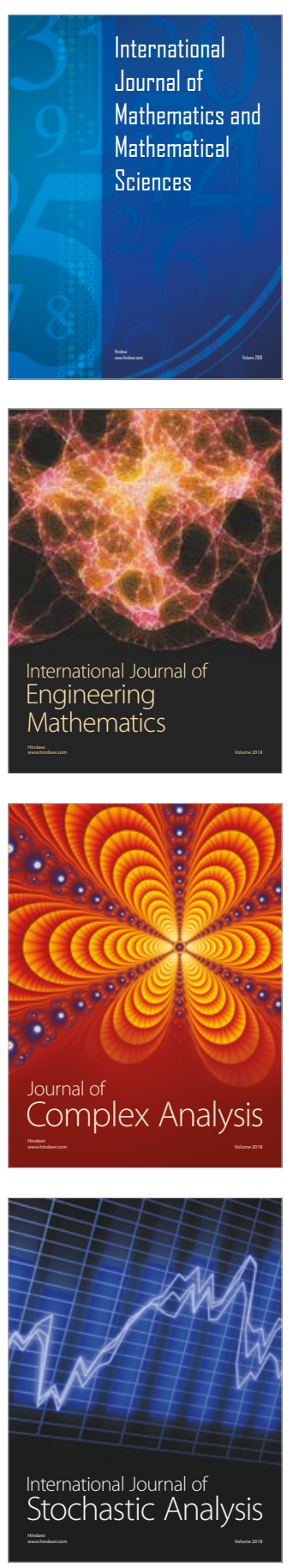
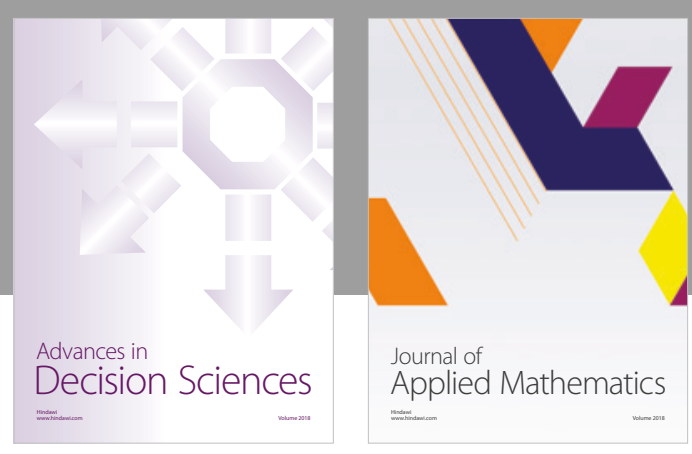

Journal of

Applied Mathematics
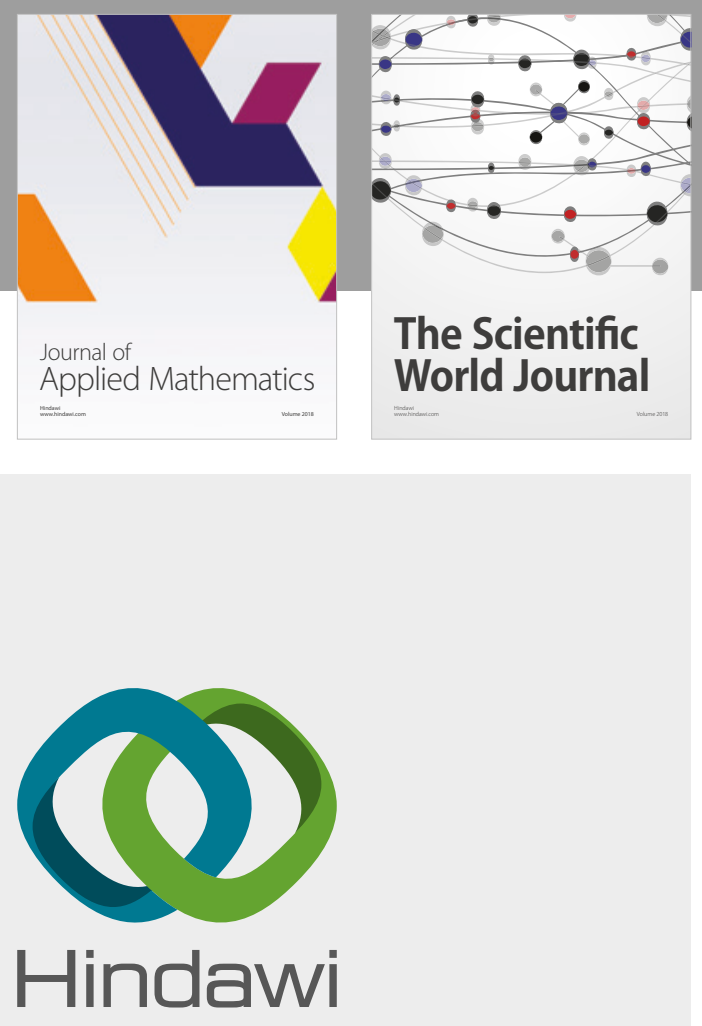

Submit your manuscripts at

www.hindawi.com

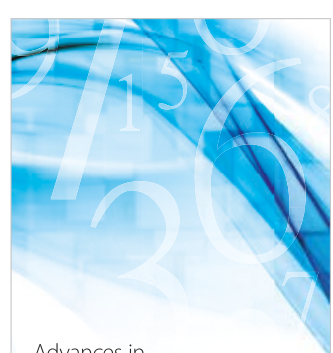

Advances in
Numerical Analysis
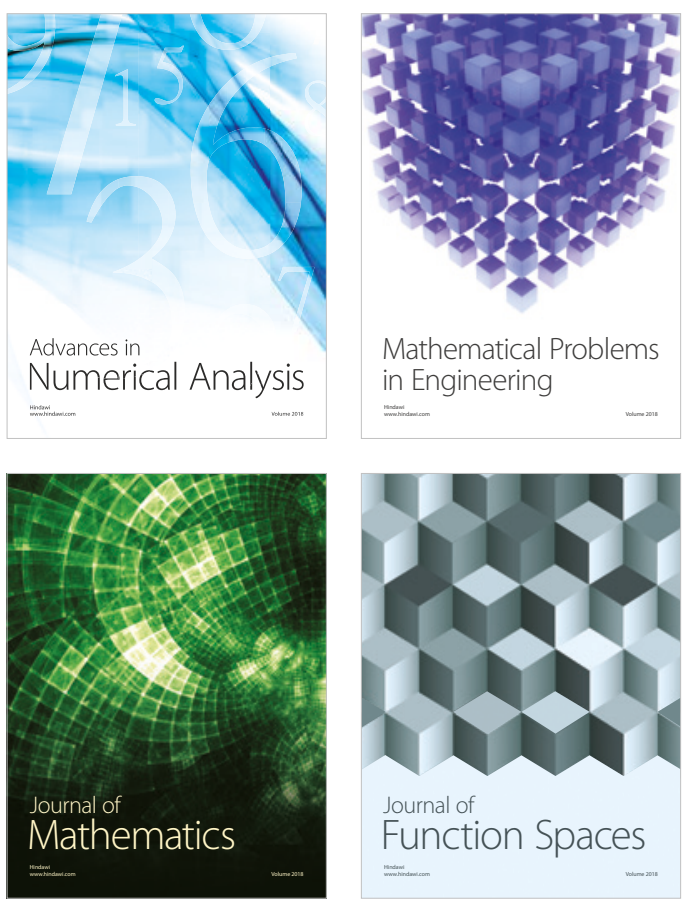

Mathematical Problems in Engineering

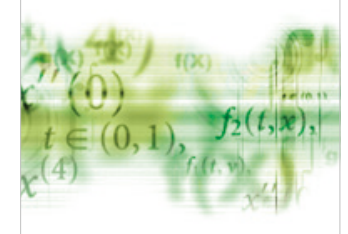

International Journal of

Differential Equations

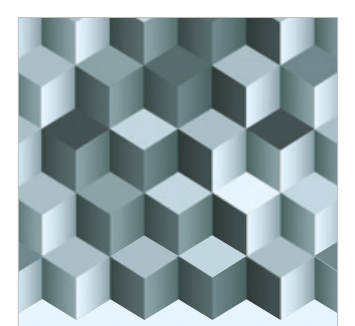

Journal of

Function Spaces

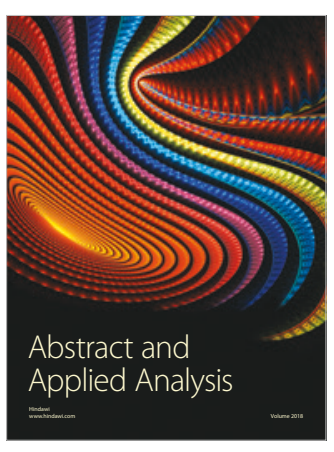

The Scientific

World Journal

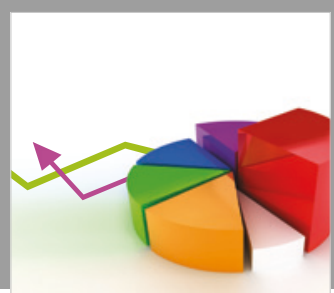

Journal of

Probability and Statistics
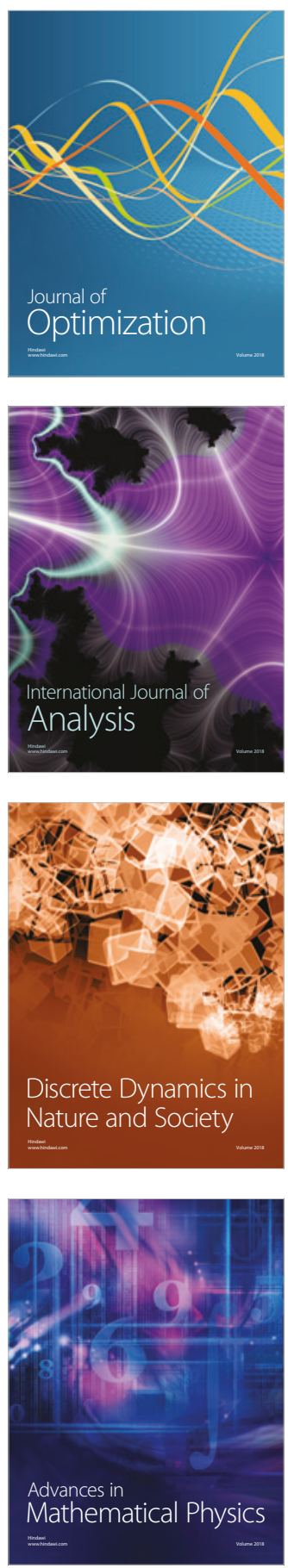